\title{
Magnoflorine Ameliorates Lipopolysaccharide-Induced Acute Lung Injury via Suppressing NF-кB and MAPK Activation
}

OPEN ACCESS

Edited by:

Patrizia Ballerini,

Università degli Studi G. d'Annunzio

Chieti e Pescara, Italy

Reviewed by:

Claudio Ferrante,

Università degli Studi G. d'Annunzio

Chieti e Pescara, Italy

Luigi Brunetti,

Università degli Studi G. d'Annunzio

Chieti e Pescara, Italy Lina Lim,

National University of Singapore,

Singapore

*Correspondence:

Ganzhen Deng

ganzhendeng@sohu.com

tThese authors have contributed

equally to this work

Specialty section:

This article was submitted to Inflammation Pharmacology,

a section of the journal

Frontiers in Pharmacology

Received: 04 May 2018 Accepted: 10 August 2018 Published: 30 August 2018

Citation:

Guo S, Jiang $K$, Wu H, Yang $C$, Yang $Y$, Yang J, Zhao $G$ and Deng $G$

(2018) Magnoflorine Ameliorates Lipopolysaccharide-Induced Acute Lung Injury via Suppressing NF- $\kappa B$ and MAPK Activation.

Front. Pharmacol. 9:982. doi: 10.3389/fphar.2018.00982

\section{Shuai Guo ${ }^{\dagger}$ Kangfeng Jiang ${ }^{\dagger}$, Haichong Wut, Chao Yang, Yaping Yang, Jing Yang, Gan Zhao and Ganzhen Deng*}

Department of Clinical Veterinary Medicine, College of Veterinary Medicine, Huazhong Agricultural University, Wuhan, China

Acute lung injury (ALI) which is featured by a strong pulmonary inflammation, is a major cause of morbidity and mortality in critically ill patients. Magnoflorine, a quaternary alkaloid isolated from Chinese herb Magnolia or Aristolochia, has been reported to have potent anti-inflammatory properties. However, the effect of magnoflorine on lipopolysaccharide (LPS)-induced ALI in mice has not been reported. The purpose of the present study is to investigate the anti-inflammatory effect of magnoflorine on LPS-induced ALI and elucidate its possible molecular mechanisms in RAW264.7 cells. The results of histopathological changes as well as the myeloperoxidase (MPO) activity indicated that magnoflorine significantly alleviated the lung injury induced by LPS. In addition, qPCR results showed that magnoflorine dose-dependently decreased the expression of pro-inflammatory cytokines TNF- $\alpha, \mathrm{IL}-1 \beta$, and IL-6. Immunofluorescence assay also confirmed that the level of Toll-like receptor 4 (TLR4) induced by LPS was inhibited by magnoflorine treatment. Further experiments were performed using Western blotting to detect the expression of related proteins in the NF- $\mathrm{kB}$ and MAPK signaling pathways. The results showed that magnoflorine suppressed the levels of phosphorylated p65, ІкB $\alpha, \mathrm{p} 38$, ERK, and JNK. In conclusion, all data indicate that magnoflorine could protect against LPS-induced inflammation in ALI at least partially by inhibiting TLR4-mediated NF-кB and MAPK signaling pathways.

Keywords: magnoflorine, anti-inflammation, ALI, LPS, NF-кB, MAPK

\section{INTRODUCTION}

Acute lung injury (ALI) is a serious respiratory disease worldwide, often accompanied by symptoms of sepsis, neutrophilia, and lung inflammation (Beutz and Abraham, 2005; Matthay and Zimmerman, 2005). It is usually caused by bacteria, trauma, and pneumonia (Treggiari et al., 2004; Lim et al., 2007). Interestingly, different mechanisms are involved in the pathogenesis of ALI. Inflammation is one of the major pathogenic factors. Although the knowledge and pharmacological therapy of ALI have developed in recent decades, the mortality rate remains high (Klugman, 1990).

Lipopolysaccharide, an endotoxin released from dead Gram-negative bacteria (Wang and Quinn, 2010), could cause leukocytosis, diffuse intravascular coagulation, and endotoxic shock, which is one of the most widely used groups of stimulants in inducing ALI in mice (Takeuchi and Akira, 2010; Lu et al., 2016). TLR4 is a transmembrane protein encoded by the TLR4 gene, which is 
involved in the innate immune response (Takeda and Akira, 2001). There are many data indicating that LPS is the ligand of TLR4 and stimulates the inflammatory response of the lungs by binding to TLR4 (Wu et al., 2016c). It is now well established that a variety of pro-inflammatory cytokines are activated by TLR4mediated NF- $\kappa$ B and MAPK signaling pathways (Xu et al., 2014; Jiang et al., 2017a). Subsequently, TNF $\alpha$, IL-1 $\beta$, IL-6, and other pro-inflammatory cytokines expression levels will be significantly increased (Yang et al., 2018). Therefore, blockade of TLR4mediated NF- $\mathrm{B}$ and MAPK signaling pathways can inhibit the development of ALI induced by LPS.

Magnoflorine, a quaternary alkaloid isolated from Chinese herb Magnolia (Nakano, 1954) or Aristolochia
(Li and Wang, 2014), has been reported to have many biological activities, such as anti-anxiety, anti-cancer, and anti-inflammation. However, the effect of magnoflorine on LPS-induced ALI in mice has not been investigated. It has been reported that the effects of LPS on ALI can be reduced by blocking various aspects of the inflammatory cascades (Shang et al., 2010; Gong et al., 2012), indicating that magnoflorine can be used as a potential drug for the treatment of ALI. In this current research, we explored whether magnoflorine could exert its anti-inflammatory action on LPS-induced ALI in mice and in RAW264.7 cells by inhibiting the NF- $\kappa$ B and MAPK signaling pathways. Importantly, the results of this study can provide some reference value for the treatment of ALI in humans.

A

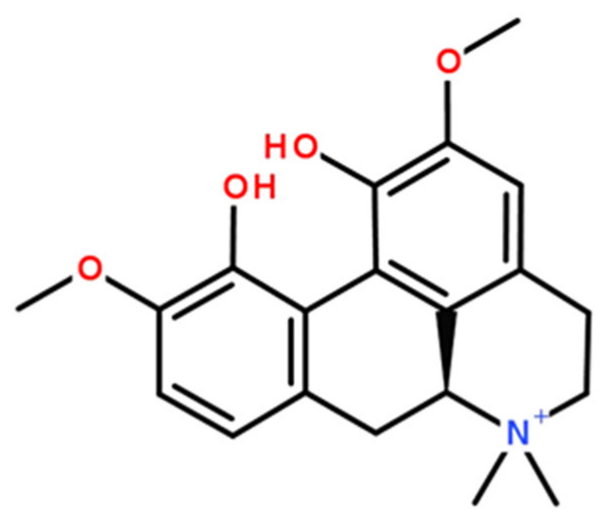

B

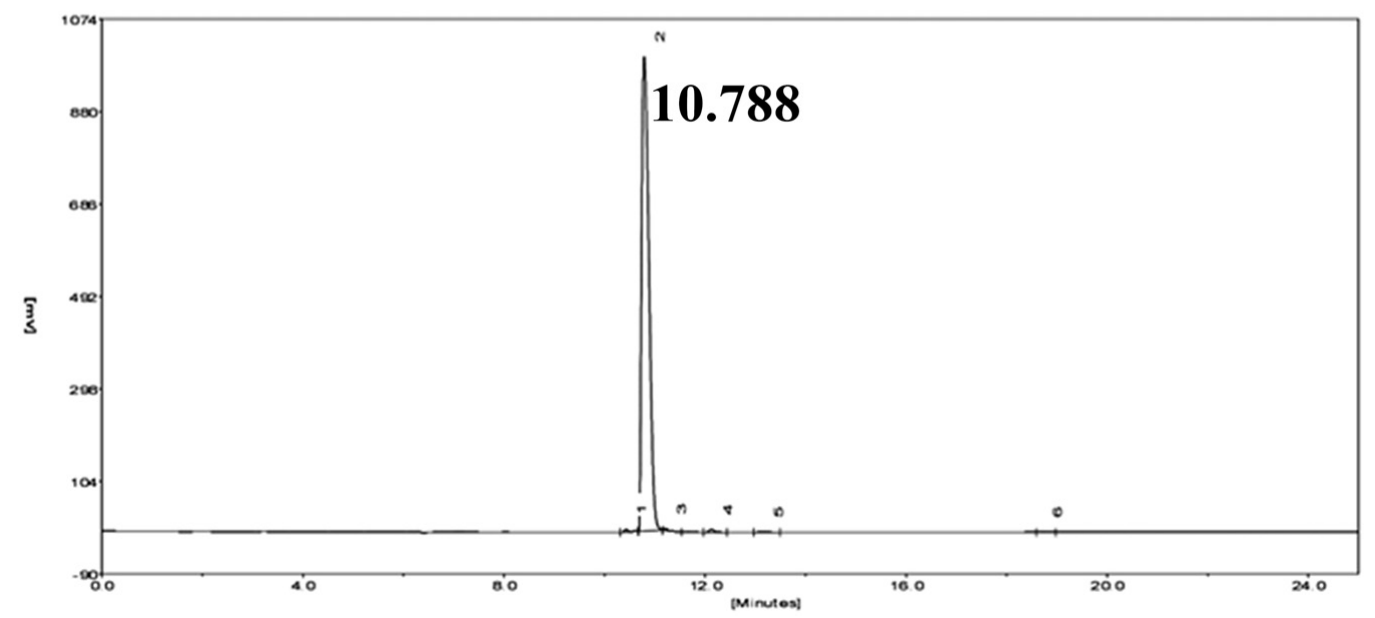

FIGURE 1 | (A) Chemical structure of magnoflorine. (B) HPLC chromatogram of magnoflorine.

TABLE 1 | Primers Used for qPCR.

\begin{tabular}{|c|c|c|c|}
\hline Name & Primer sequence $\left(5^{\prime}-3^{\prime}\right)$ & GenBank accession number & Product size (bp) \\
\hline TNF- $\alpha$ & CTTCTCATTCCTGCTTGTG ACTTGGTGGTITGCTACG & NM_013693.3 & 198 \\
\hline IL-1 $\beta$ & CCTGGGCTGTCCTGATGAGAG TCCACGGGAAAGACACAGGTA & NM_008361.4 & 131 \\
\hline IL-6 & GGCGGATCGGATGTTGTGAT GGACCCCAGACAATCGGTTG & NM_031168.1 & 199 \\
\hline GAPDH & CAATGTGTCCGTCGTGGATCT GTCCTCAGTGTAGCCCAAGATG & NM_001289726.1 & 124 \\
\hline
\end{tabular}



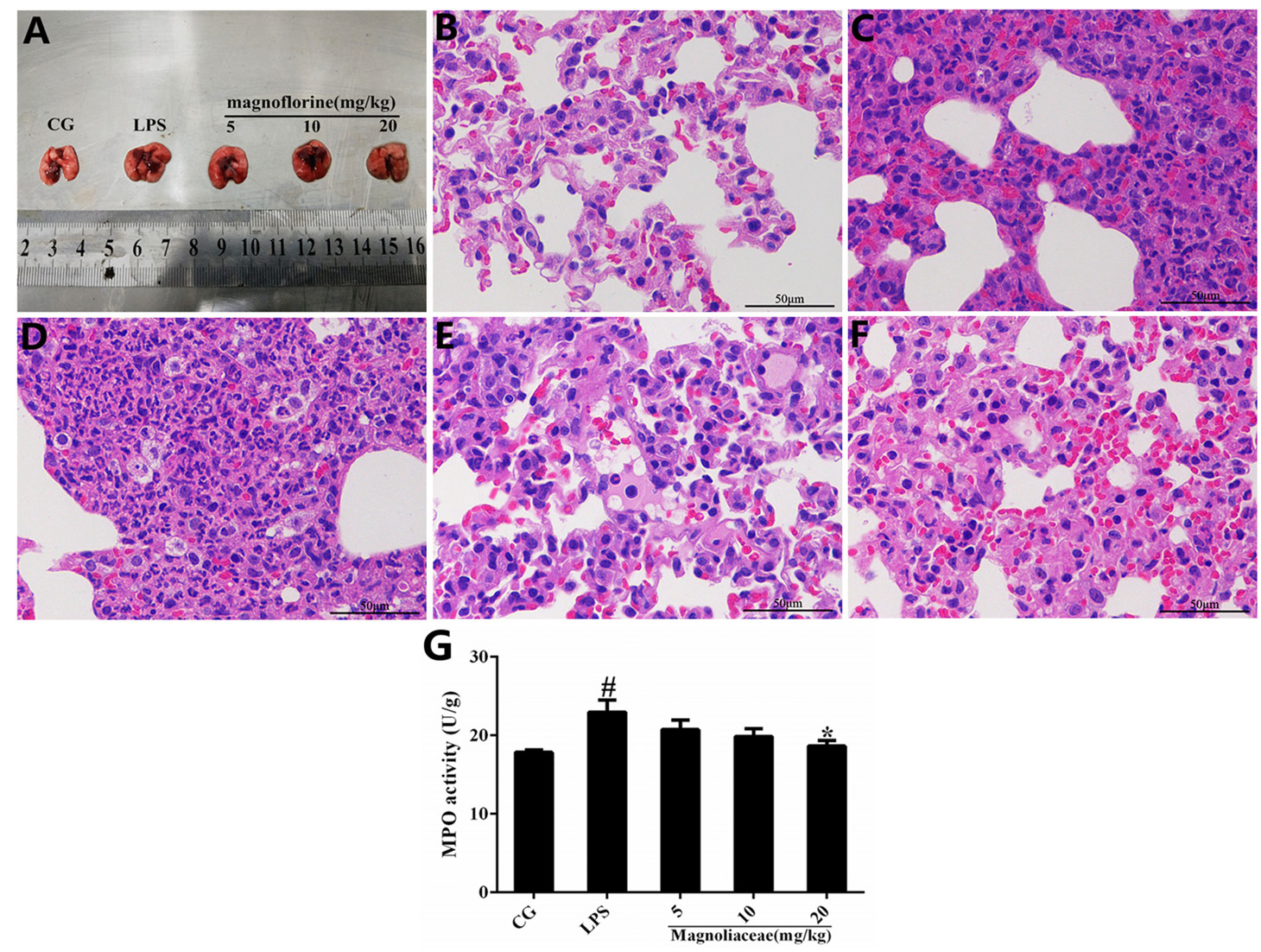

FIGURE 2 | Effects of magnoflorine on LPS-induced lung injury. (A) Morphology of the lung. (B) Control group. (C) LPS group. (D-F) Magnoflorine (5, 10, and $20 \mathrm{mg} / \mathrm{kg}$ ) groups. (G). MPO activity assay. CG is the control group. LPS is the LPS-stimulated group. The values are presented as means \pm S.E.M. of three independent experiments. ANOVA, $p<0.0001$, post hoc ${ }^{*} p<0.05$ vs. control group, ${ }^{*} p<0.05$ vs. LPS group.

\section{MATERIALS AND METHODS}

\section{Reagents}

Magnoflorine (HPLC $\geq 98 \%$ ) was obtained from Shanghai Yuanye Biotechnology Co., Ltd. (Shanghai, China) (Figure 1). LPS (Escherichia coli 055:B5) was purchased from Sigma (St. Louis, MO, United States). The myeloperoxidase (MPO) determination kits were provided by the Jiancheng Bioengineering Institute of Nanjing (Nanjing, China). The qPCR kit was obtained from Takara Bio Inc., (Otsu, Japan). NF-кB and MAPK antibodies were purchased from Cell Signaling Technology (Danvers, MA). All other chemical reagents were in accordance with the reagent specification level. All other chemical reagents meet the reagent specification standards.

\section{Animal Treatment and Experimental Groups}

A total of $50 \mathrm{BALB} / \mathrm{c}$ male mices (6-8 weeks old, 30-35 g weight) were purchased from Wuhan Institute of Biological Products Co., Ltd. (Wuhan, China). All mices are kept in the special environment of $24^{\circ} \mathrm{C} \pm 1^{\circ} \mathrm{C}$, and $65 \%$ humidity, which maintain $12 \mathrm{~h}$ of light for 3 days to adapt to the environment before starting the experiments. During the trial, all animals were

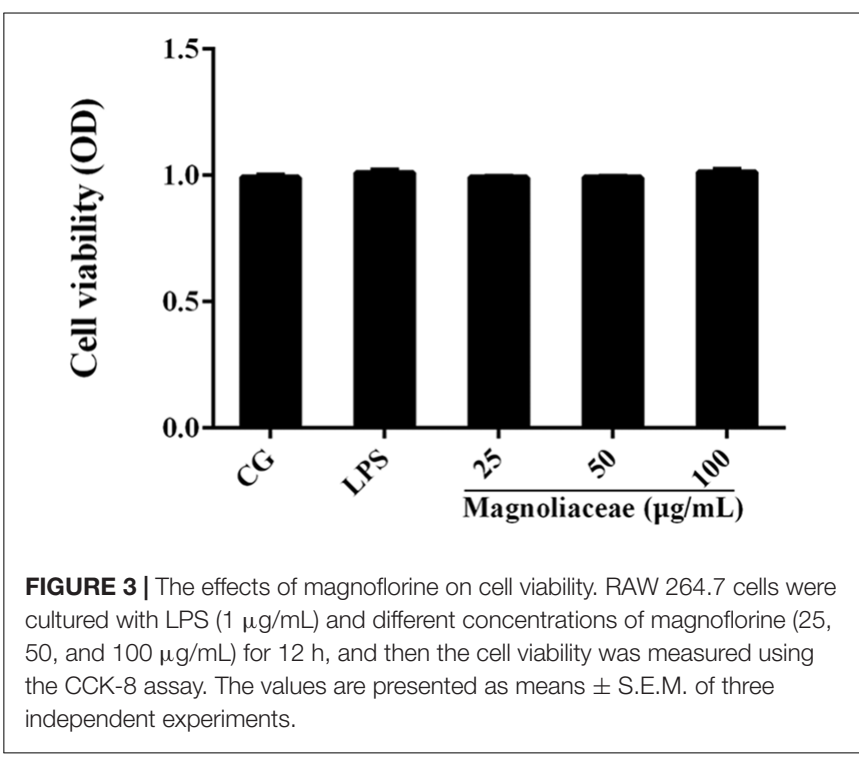

allowed to drink and feed ad libitum. This study was carried out in accordance with guidelines provided by the Laboratory Animal Research Center of Hubei province, and approved 
by the Ethical Committee on Animal Research at Huazhong Agricultural University (HZAUMO-2015-12).

The mouse were randomly divided into the following five groups of ten mice in each group for the establishment of ALI model:blank group, LPS group, Magnoflorine (5, 10, and $20 \mathrm{mg} / \mathrm{kg}$ ) + LPS groups. Magnoflorine was diluted with Dulbecco's modified Eagle's medium (DMEM) to different concentrations. LPS was diluted with phosphate buffered saline (PBS) to a final concentration of $1 \mathrm{mg} / \mathrm{ml}$. The method for establishing the LPS-induced ALI model was described previously (Li and Wang, 2014). Briefly, the mice were intranasally administered $50 \mu \mathrm{L}$ of LPS to induce ALI. The blank group was intranasally administered $50 \mu \mathrm{L}$ of PBS. After $24 \mathrm{~h}$ of instillation, The mice in the magnoflorine group were intraperitoneally injected with different concentrations of magnoflorine $(5,10$, and $20 \mathrm{mg} / \mathrm{kg})$ three times at 0,8 , $16 \mathrm{~h}$. The blank group received equal volumes of PBS. $8 \mathrm{~h}$ after the last treatment with magnoflorine, the mice were were

\section{A \\ (Tissue)}
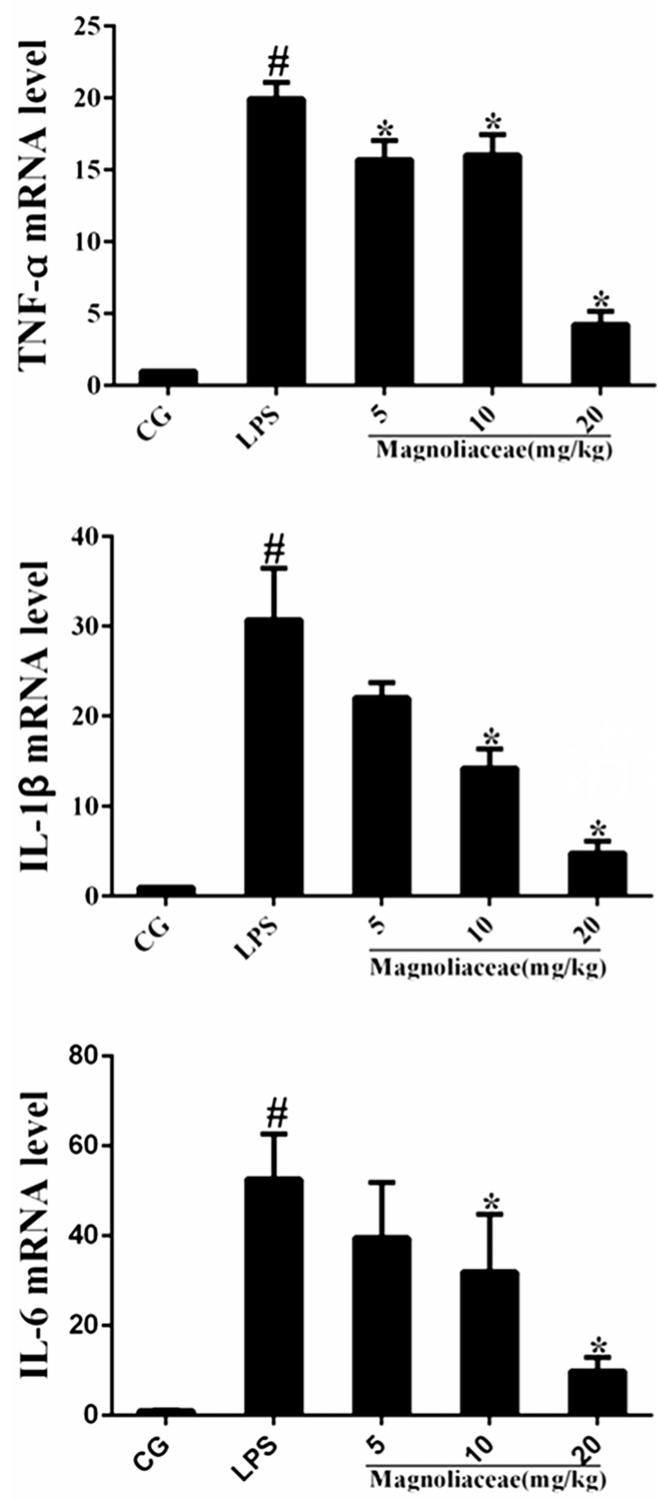

(Cell)
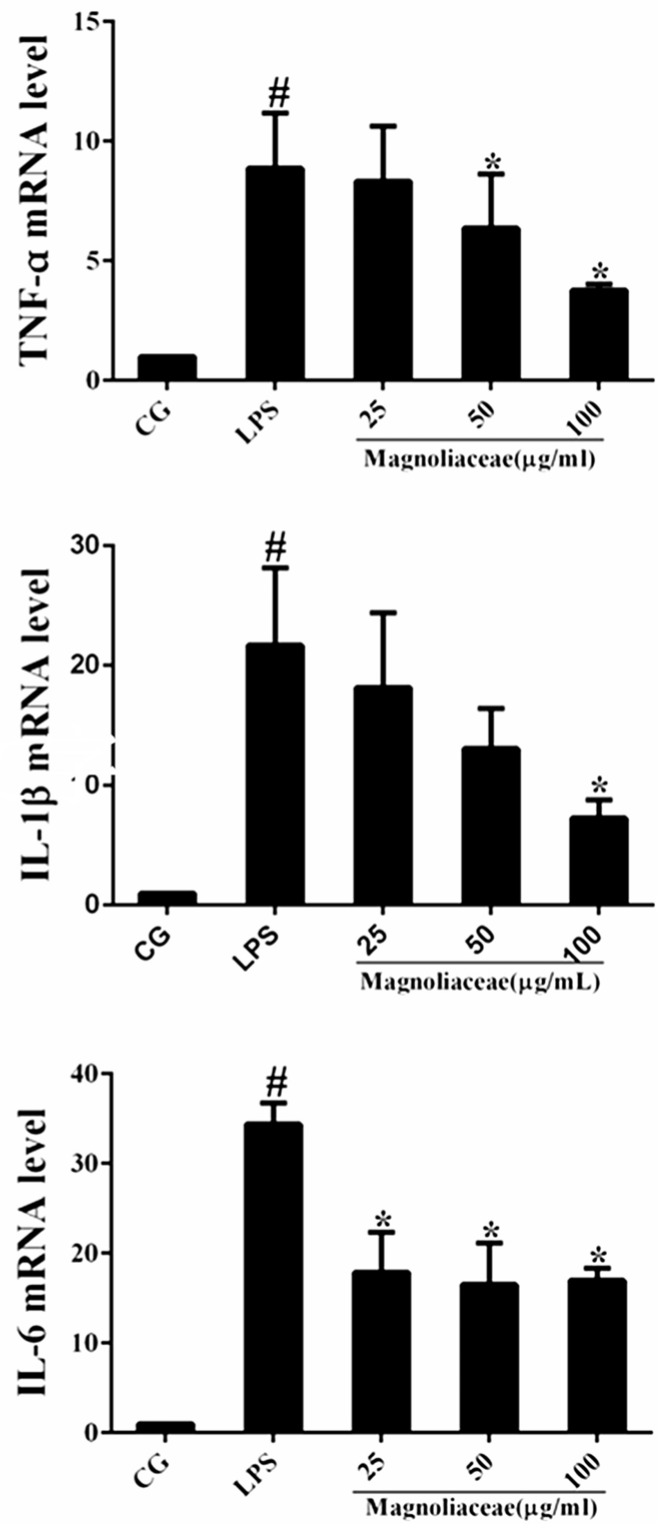

FIGURE 4 | Effects of magnoflorine on the production of cytokines. (A) The expression of TNF- $\alpha$, IL-1 $\beta$, and IL-6 mRNA in lung tissues were measured by qPCR. (B) The expression of TNF- $\alpha$, IL-1 $\beta$, and IL-6 mRNA in RAW264.7cells were measured by qPCR. GAPDH was used as a control. CG is the control group. LPS is the LPS-stimulated group. The data are presented as the mean \pm S.E.M. of three independent experiments. ANOVA, $p<0.0001, p 0 s t$ hoc ${ }^{\#} p<0.05$ vs. control group, $* p<0.05$ vs. LPS group. 
euthanized, and the lung tissue were harvested and kept at $-80^{\circ} \mathrm{C}$.

\section{High-Performance Liquid Chromatography (HPLC)}

The purity of magnoflorine was measured by HPLC. The experiment was carried out using an EChrom2000 DAD data system (Elite, Dalian, China) as described previously (Wu et al., 2016d). Briefly, the separation was performed on a Hypersil ODS2-C18 analytical column $(5 \mu \mathrm{m}, 200 \mathrm{~mm} \times 4.6 \mathrm{~mm})$. Subsequently, the elution was performed using the acetonitrilewater $(2: 98, \mathrm{v} / \mathrm{v})$ mobile phase. The flow rate was $1.0 \mathrm{~mL} / \mathrm{min}$, and the detection wavelength was $268 \mathrm{~nm}$.

\section{Histopathologic Evaluation of the Lung Tissue}

Lung tissues were obtained, cut into sections of approximately $0.5 \mathrm{~cm}^{2}$ sizes, and fixed in $10 \%$ formalin for subsequent histopathological analysis. Briefly, lung tissues were dehydrated with different concentrations of alcohol, infiltrated with xylene, embedded in paraffin, and sliced into $4 \mu \mathrm{m}$ sections, and then stained with hematoxylin-eosin (H\&E). Finally, the morphology changes of lung tissues were observed by optical microscope (Olympus).

\section{Myeloperoxidase Analysis}

The level of MPO activity can be used to predict the early risk of inflammatory diseases (Li et al., 2015). Lung tissue was collected and ground into a tissue homogenate with a reaction buffer (w/v, 1/19), after which MPO activity was detected and analyzed according to the instructions of manufacturer's MPO assay kit.

\section{Cell Viability Assay}

A Cell Counting Kit-8 (CCK-8) was used for the determination of cell viability. RAW264.7 cells were grown at a density of $2 \times 10^{4}$ cells $/ \mathrm{mL}$ in 96 well plates. After the cells were adherent (approximately $2 \mathrm{~h}$ ), the cells were treated with different concentrations of magnoflorine $(25,50,100 \mu \mathrm{g} / \mathrm{mL})$. After $12 \mathrm{~h}$, $10 \mu \mathrm{L}$ of CCK-8 was added in each well for $4 \mathrm{~h}$ at $37^{\circ} \mathrm{C}$. And the $\mathrm{OD}$ value of the cells in each well was measured at $450 \mathrm{~nm}$ with a microplate reader. The cell viability $=($ Treatment Group OD Blank Group OD)/(Control Group OD - Blank Group OD).

\section{Cell Culture and Treatment}

RAW264.7 cells were purchased from the American Type Culture Collection (ATCC TIB-71 ${ }^{\mathrm{TM}}$ ). The cells were cultured in DMEM medium supplemented with $10 \%$ fetal bovine serum at $37^{\circ} \mathrm{C}$ with $5 \% \mathrm{CO} 2$. The cells were pretreated with various concentrations of magnoflorine $(25,50$, and $100 \mu \mathrm{g} / \mathrm{mL})$ for $1 \mathrm{~h}$ and then stimulated with LPS $(1 \mu \mathrm{g} / \mu \mathrm{L})$ for $12 \mathrm{~h}$. The cells that were not given any treatment were used as a control group.

\section{Quantitative PCR Assay}

According to the manufacturer's instructions, total RNA was extracted from tissues and cells using the Trizol reagent, and then cDNA was generated using a reverse transcription kit (Takara,
Japan). qPCR was performed using SYBR Green plus reagent kit (Roche, Basel, Switzerland) with Light- Cycler 96 (Roche) following the instructions of the manufacturer. The expression levels of inflammatory genes were normalized to GAPDH with $2^{-\Delta \Delta C_{\mathrm{t}}}$ method as described previously (Livak and Schmittgen, 2001). The primers used for qPCR are listed in Table $\mathbf{1}$.

\section{Immunofluorescence Staining}

RAW264.7 cells $\left(1 \times 10^{5}\right.$ cells $\left.\mathrm{mL}^{-1}\right)$ were seeded onto a six-wellplate and then were pretreated with various concentrations of magnoflorine $(25,50$, and $100 \mu \mathrm{g} / \mathrm{mL})$ for $1 \mathrm{~h}$ and then stimulated with LPS $(1 \mu \mathrm{g} / \mu \mathrm{L})$ for $12 \mathrm{~h}$. The cells were fixed with $4 \%$ paraformaldehyde for $10 \mathrm{~min}$, permeabilized with $0.2 \%$ Triton X-100 for $10 \mathrm{~min}$, blocked with 5\% BSA for $1 \mathrm{~h}$ and followed by incubation with rabbit anti-p-p65 antibody and anti-TLR4 antibody overnight at $4^{\circ} \mathrm{C}$. Subsequently, the cells were washed and incubated with FITC-labeled goat anti-rabbit IgG antibody for $1 \mathrm{~h}$. Nuclei were stained with DAPI for $10 \mathrm{~min}$, and the p-p65 and TLR4 were observed using a fluorescence microscope (Olympus, Japan).

\section{Western Blot Analysis}

Lung tissues and RAW264.7 cells were lysed with a lysate containing a phosphatase inhibitor and then centrifuged at $4^{\circ} \mathrm{C}$ and $12,000 \mathrm{rpm}$ for $15 \mathrm{~min}$. The obtained protein was measured for its concentration by a Biosharp protein measuring kit. Subsequently, sodium dodecyl sulphonate polyacrylamide gel electrophoresis was performed and $40 \mu \mathrm{g}$ protein was loaded per well (at the same concentration). The separated protein was

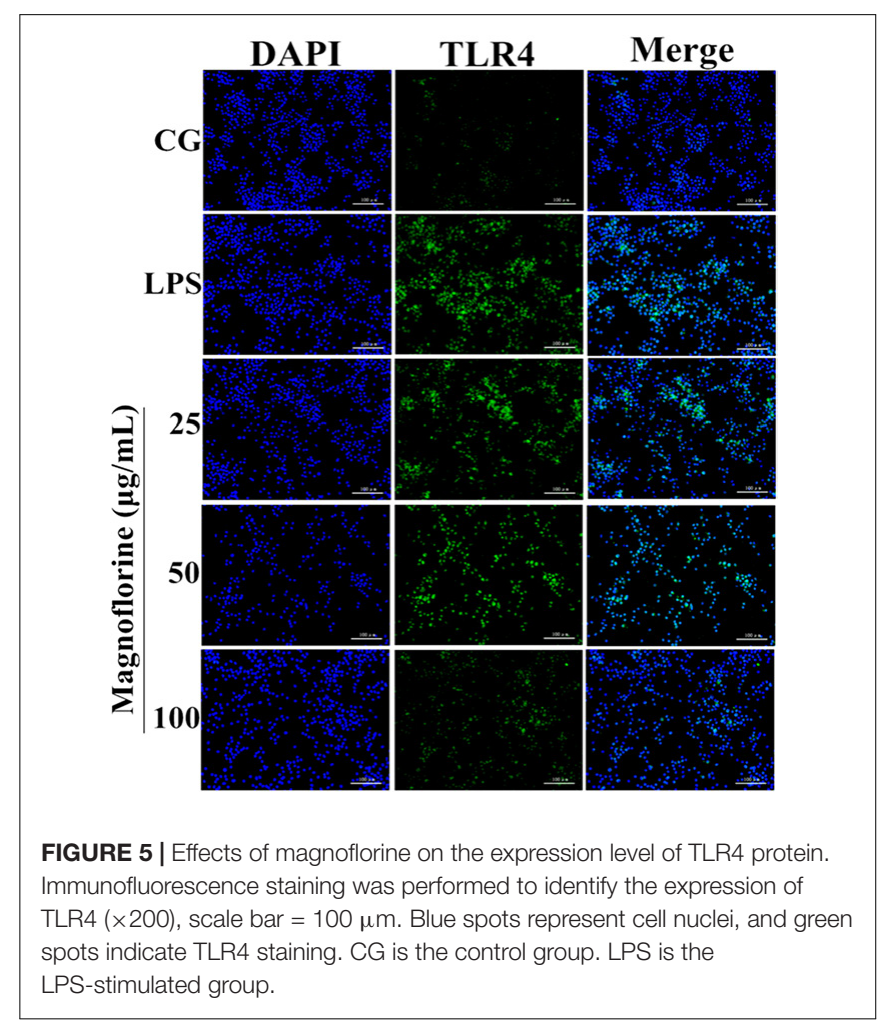




\section{A \\ (Tissue)}
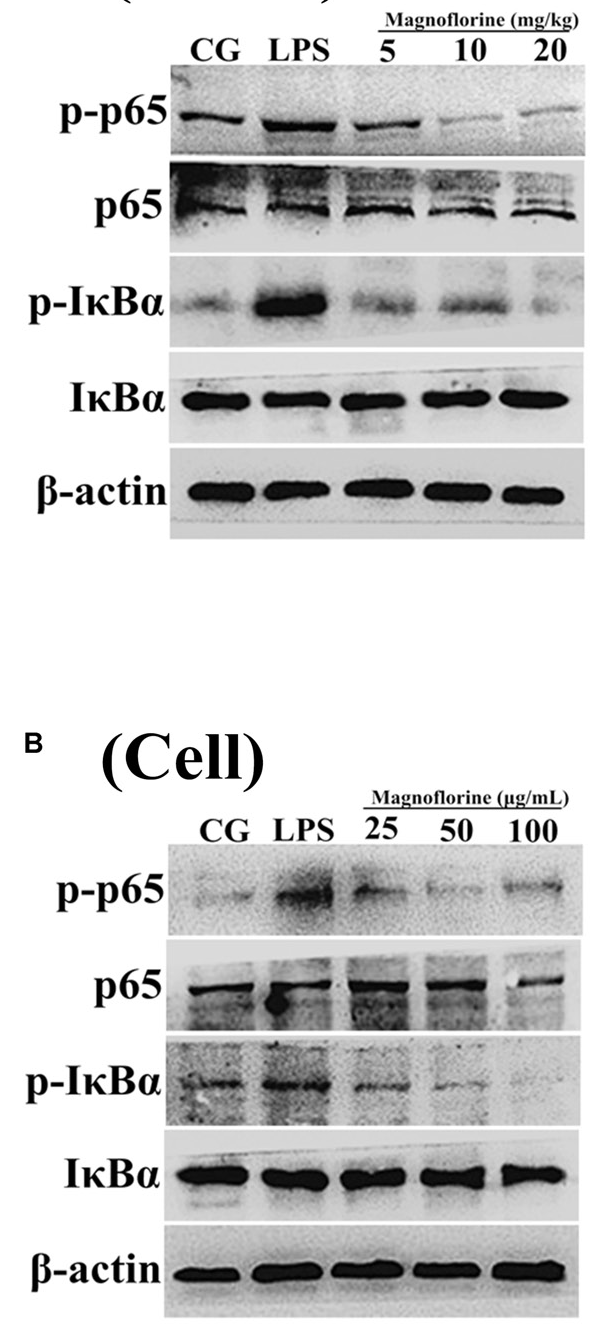
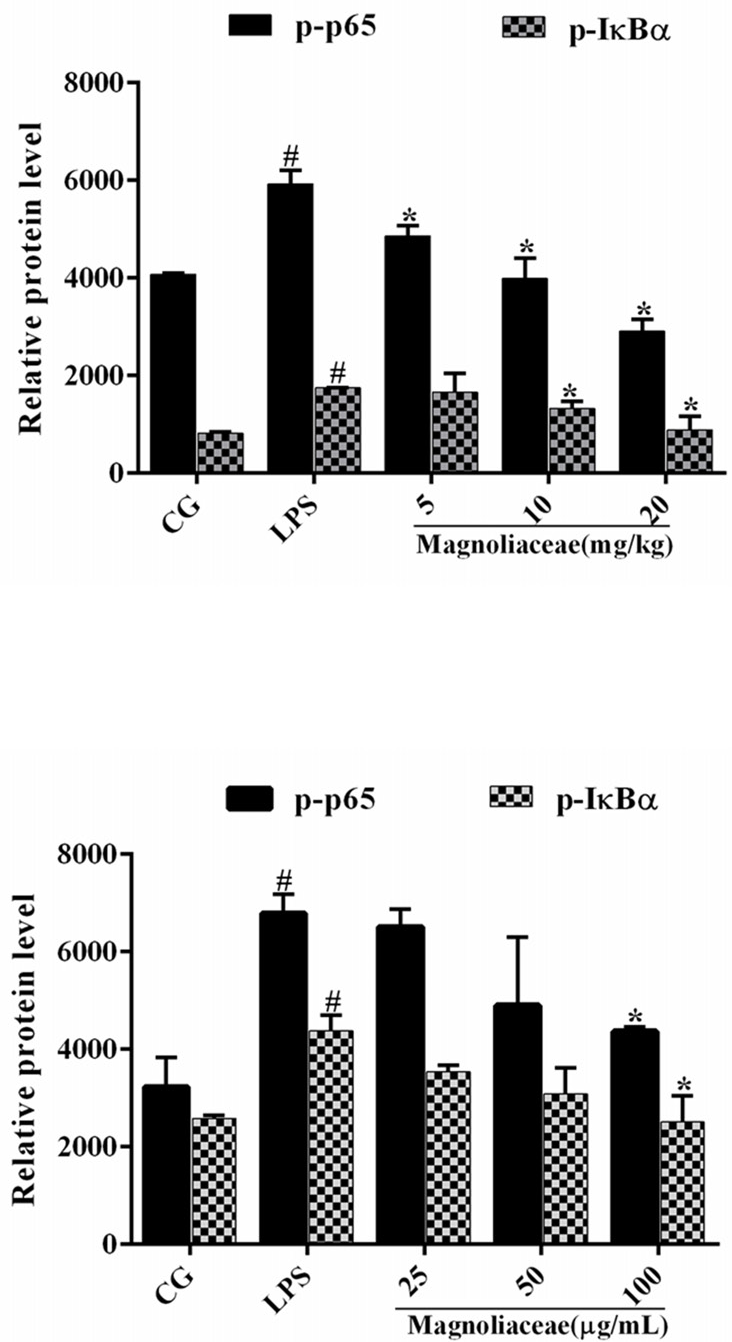

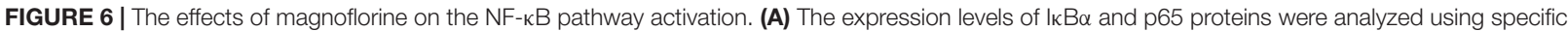
antibodies in lung tissues. (B) The expression levels of $I_{\kappa} B \alpha$ and p65 proteins in RAW264.7 cells. $\beta$-actin was used as the control. CG is the control group. LPS is the LPS-stimulated group. The data represent the mean \pm S.E.M. ANOVA, $p<0.0001$, post hoc ${ }^{\#} p<0.05$ vs. control group, ${ }^{*} p<0.05$ vs. LPS group.

transferred to polyvinylidene difluoride membrane and blocked in blocking solution for $2 \mathrm{~h}$, and then incubated overnight at $4^{\circ} \mathrm{C}$ with primary antibodies (1:1000). Afterward, the membranes were incubated with secondary antibodies (1:4000) for $1 \mathrm{~h}$ at $25^{\circ} \mathrm{C}$. The protein levels were detected with an enhanced chemiluminescence reagent, and the intensities were quantified using Image J gel analysis software.

\section{Statistical Analyses}

The SPSS software 16.0 (SPSS Inc.) was used for the statistical analyses. Statistical data were expressed as the mean \pm SEM of three individual experiments. The data were analyzed using ANOVA followed by Dunnet's post hoc test. $P$-values less than 0.05 were deemed statistically significant differences.

\section{RESULTS}

\section{Effects of Magnoflorine on LPS-Induced Lung Injury in Mice}

Histopathological analysis and MPO assay were used to determine lung tissue damage (Figure 2A). There were no histopathological lesions in the control group (Figure 2B), whereas pathological changes such as infiltration of inflammatory cells and alveolar hyperemia were observed in the LPS group (Figure 2C). Interestingly, compared with the LPS group, the infiltration of inflammatory cells and the extent of alveolar congestion were significantly reduced in the magnoflorine groups (Figures 2C-F). A further MPO test was also used to analyze the effect of magnoflorine on LPS-induced lung injury. The results showed that LPS dramatically increased MPO activity, 
which was significantly reduced with magnoflorine treatment (Figure 2G).

\section{Effects of Magnoflorine on Cell Viability}

The potential cytotoxicity of magnoflorine on RAW264.7 cells was determined using the CCK- 8 assay. The results show that magnoflorine has no effect on cell viability (Figure 3).

\section{Effects of Magnoflorine on the Levels of Cytokines}

The expression levels of inflammatory cytokines in lung tissue and RAW264.7 cells were examined by qPCR. The results of the qPCR assay showed that the expression levels of TNF- $\alpha$, IL- $1 \beta$, and IL- 6 in the LPS group were significantly higher than those in the control group. The expression levels of the three inflammatory factors in the magnoflorine group were dosedependently reduced compared to the LPS group (Figures 4A,B).

\section{Magnoflorine Inhibition of the Expression of TLR4}

TLR4 is the first TLR receptor protein to play a role in the LPS reaction (Jiang et al., 2018), which is of great significance in LPSinduced ALI. As shown by Immunofluorescence assay, LPS group significantly increased TLR4 expression. However, the expression levels of TLR4 protein were decreased by magnoflorine groups (Figure 5).

\section{Effects of Magnoflorine on the NF-кB Pathway in LPS-Induced ALI}

$\mathrm{NF}-\kappa \mathrm{B}$ signaling pathway is one of the important signaling pathways of inflammatory response. In order to further test the effect of magnoflorine on LPS-induced NF- $\kappa \mathrm{B}$ signaling pathway, the expression of $\mathrm{NF}-\kappa \mathrm{B}$ p 65 and $\mathrm{I} \kappa \mathrm{B} \alpha$ protein was detected by Western blot. The results showed that the expression of phosphorylated p65 and Iк $\mathrm{B} \alpha$ protein in the lung tissue was significantly higher than that in the control group. Interestingly, the expression of magnoflorine groups were relatively reduced (Figure 6A). Furthermore, in RAW264.7 cells, the expression levels of phosphorylated $\mathrm{p} 65$ and $\mathrm{I} \kappa \mathrm{B} \alpha$ proteins were significantly higher than those in the control group, whereas the expression of the magnoflorine protein decreased in a dose-dependent manner (Figure 6B). To further confirm these observations, We examined the nuclear translocation of $\mathrm{p} 65$ protein in RAW264.7 cells. We found that the expression of nuclear p65 was significantly reduced after treatment with magnoflorine (Figure 7).

\section{Effects of Magnoflorine on the MAPK Pathway in LPS-Induced ALI}

Compared with NF- $\kappa \mathrm{B}, \mathrm{MAPK}$ is also a very important signaling pathway. The inhibitory effect of magnoflorine on the MAPK signaling pathway was evaluated by measuring the expression levels of p38, ERK and JNK proteins. The results showed that in the lung tissue, the expression of phosphorylated p38, ERK, and JNK proteins was significantly increased in the
LPS group compared with the control group. In contrast, the expression levels of phosphorylated p38, ERK, and JNK proteins in the magnoflorine groups were dose-dependently lower than the LPS group (Figure 8A). In addition, in RAW264.7 cells, LPS phosphorylated p38, ERK, and JNK protein expression levels were significantly higher than the control group, while the expression of magnoflorine groups were relatively reduced (Figure 8B).

\section{DISCUSSION}

Although inflammation is considered as a protective mechanism elicited by the host in answer to various aggressions such as microbial infections, excessive inflammation often causes extensive tissue damage and even systemic dysfunction (Kuriakose et al., 2013; Chen et al., 2015). ALI is characterized by obvious acute inflammation with elevated pro-inflammatory cytokines levels, and is a major cause of morbidity and mortality in critically ill patients (Wu et al., 2016b). Recent studies have shown that magnoflorine has a certain anti-inflammatory effect (Li and Wang, 2014). Besides, magnoflorine has also been shown to possess potent an-tiradical and an-tioxidant activities (Rackovã et al., 2004), and this feature is typically related to the secondary metabolites with free phenolic structure such as resveratrol and apigenin (Chiavaroli et al., 2010; Menghini et al., 2016b). In addition, magnoflorine also have many biological activities, such as anti-anxiety, and anti-cancer (Li and Wang, 2014). Importantly, it can protect the oxidation of human low density lipoprotein (Hung et al., 2007). However,

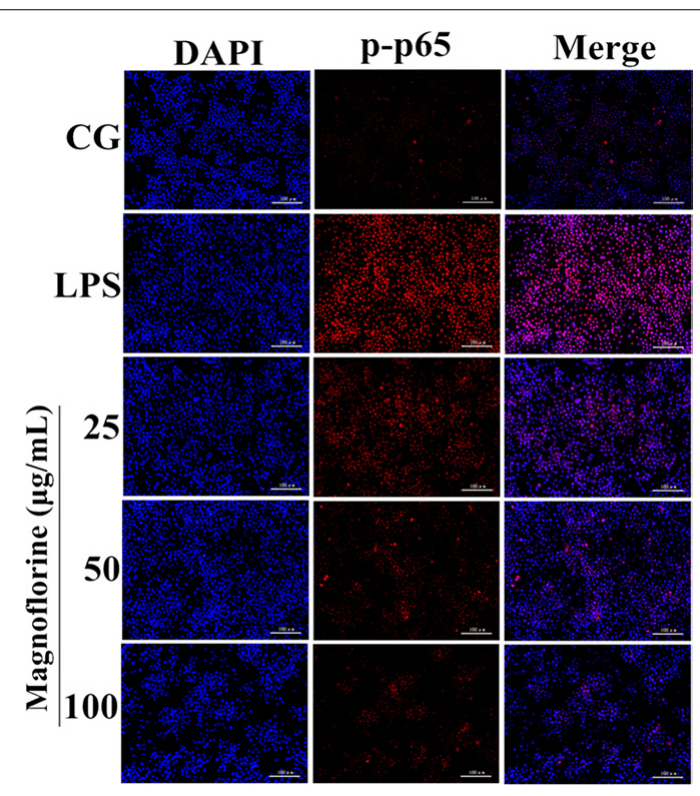

FIGURE 7 | Effects of magnoflorine on p65 translocation into the nucleus. Translocation of the p65 subunit from the cytoplasm into the nucleus was assessed by immunofluorescence staining $(\times 200)$, scale bar $=100 \mu \mathrm{m}$. Blue spots represent cell nuclei, and red spots indicate p-p65 staining. CG is the control group. LPS is the LPS-stimulated group. 


\section{A (Tissue)}

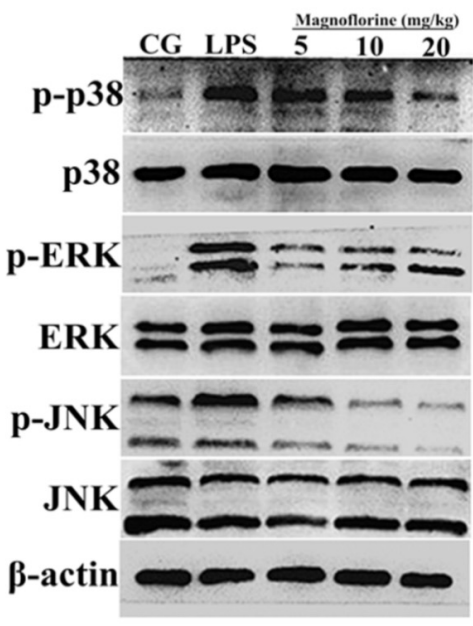

\section{B (Cell)}

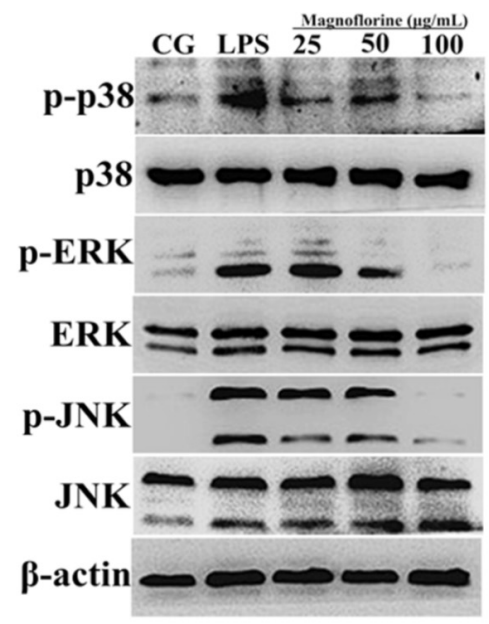

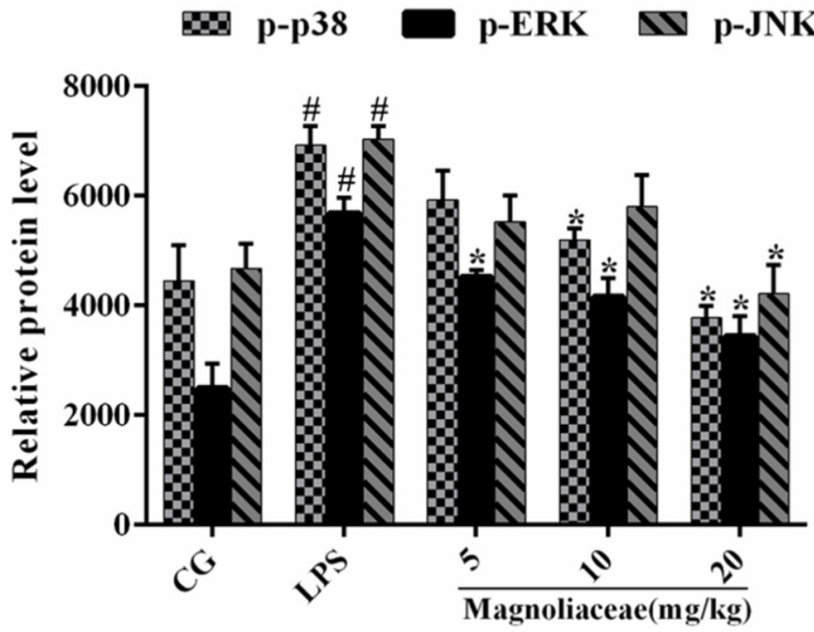

8 p-p38 p-ERK $\square$ p-JNK

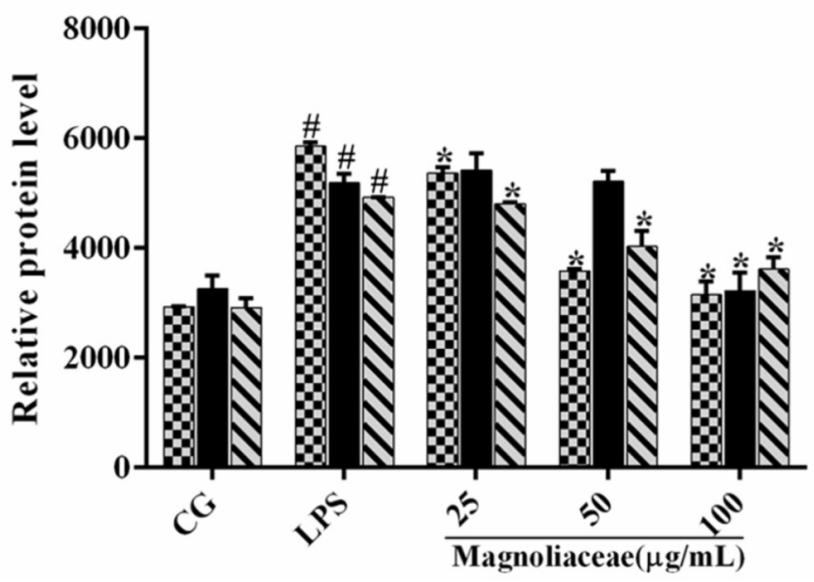

FIGURE 8 | Effects of magnoflorine on the MAPK pathway activation. (A) The expression levels of p38, ERK, and JNK proteins in lung tissues. (B) The expression levels of p38, ERK, and JNK proteins in RAW264.7 cells. $\beta$-actin was used as the control. CG is the control group. LPS is the LPS-stimulated group. The data represent the mean \pm S.E.M. ANOVA, $p<0.0001$, post hoc ${ }^{\#} p<0.05$ vs. control group, ${ }^{*} p<0.05$ vs. LPS group.

the effect of magnoflorine on LPS-induced ALI in mice has not been reported. In the present study, we investigated the anti-inflammatory effect of magnoflorine on LPS-induced ALI in vivo and in vitro.

It is well-known that inflammation can damage the normal lung structure and cause exudation of inflammatory products (Driver, 2012). Through the histopathological observation, we found that magnoflorine inhibited the infiltration of inflammatory cells and restrained the alveolar structural damage. Importantly, evidence has been increasing that oxidative stress could induce aberrant activation of macrophages and then results in inflammatory damage (Cachofeiro et al., 2008).
Hence, the radical scavenger property of magnoflorine may be a possible mechanism of action related to the observed protective effects. It has been reported that MPO is a biomarker of neutrophil migration into tissues, which can reflect the number of neutrophils in inflamed or injured tissues (Jiang et al., 2017b). Moreover, MPO as an important therapeutic target in the treatment of inflammatory conditions and its activity reflects the infiltration of neutrophils into lung tissues (Odobasic et al., 2014). The results of the MPO assay showed that magnoflorine markedly reduced MPO activity in LPS-induced ALI, suggesting that magnoflorine could repress neutrophil influx into lung tissues. As an important immune cell, RAW 


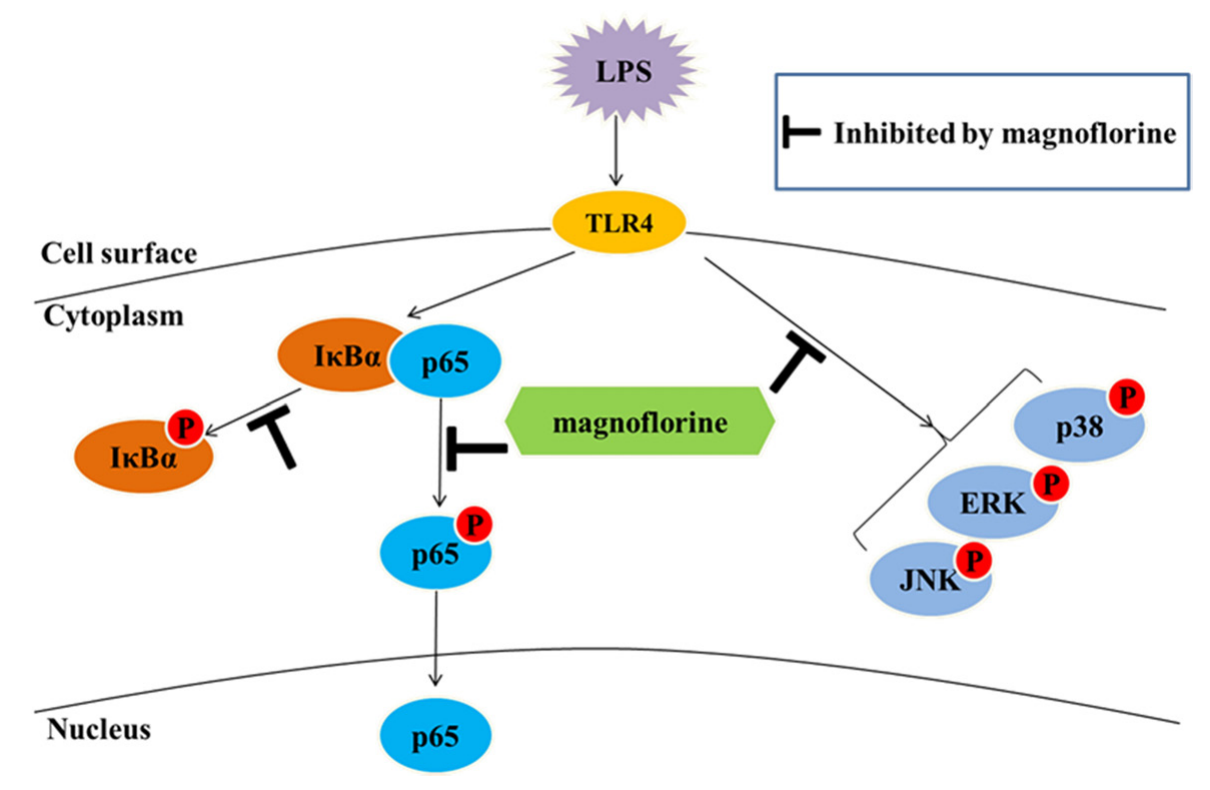

FIGURE 9 | NF-kB and MAPK pathways in LPS-stimulated ALI.

264.7 murine macrophages have been widely used in the establishment of mouse inflammation model in ALI in vitro. Thus, we explored the effects of magnoflorine on LPS-stimulated RAW264.7 cells. The macrophage is the important sensory and regulatory cell in immunological system; thus, we also examined the effect of magnoflorine on LPS-stimulated RAW264.7 macrophages. The CCK-8 assay showed that the different doses of magnoflorine have no toxicity to cells, consistent with a previous study.

Proinflammatory cytokines appear in the early stages of inflammation (Giebelen et al., 2007), which indicate the severity of ALI in a certain sense. LPS stimulation releases inflammatory cytokines such as TNF- $\alpha$, IL- $1 \beta$, IL-6, and increases their expression levels (Zhang et al., 2011). TNF- $\alpha$ is an important cytokine secreted by macrophages that promotes the activation of neutrophils and the release of other cytokines (Akira et al., 1990; Wu et al., 2015). Similar to TNF- $\alpha$, IL-1 $\beta$ is also secreted by macrophages and, to some extent, regulates the progress of the inflammatory response (Akira et al., 1990). IL-6 maintains tissue homeostasis and reflects the extent of tissue damage, which is critical in the inflammatory response (Cronin et al., 2016). In addition, IL-6 could also exert a downregulating effect on pro-inflammatory TNF $\alpha$ (Menghini et al., 2016a). In our study, the TNF- $\alpha$, IL-1 $\beta$, and IL-6 levels in lung tissues and macrophages were evidently lower in the magnoflorine groups than in the LPS group. These results revealed that magnoflorine exerted anti-inflammatory effects, perhaps by reducing the levels of pro-inflammatory cytokines.

TLR4, a member of the toll-like receptor family, plays an important role in the innate immune response (Takeda and Akira, 2004; Mateu et al., 2015). Previous reports have shown that TLR4 participates in LPS-induced immune responses by activating the $\mathrm{NF}-\kappa \mathrm{B}$ and MAPK signaling pathways (Wu et al., 2016a). To further enlighten the mechanism by which magnoflorine exerts its potent anti-inflammatory action, we then explored the TLR4-mediated activation of the NF-кB and MAPK signaling pathways. We found that LPS significantly increases the expression of TLR4, while magnoflorine treatment reduced TLR4 expression to varying degrees. It has been reported that both NF- $\mathrm{B}$ and MAPK signaling pathways are involved in LPS-induced mice ALI (Lin et al., 2018). NF- $\kappa$ B, a critical factor linking inflammation and tumorigenesis, consists of p50, p52, p65, RelB, and c-Rel, and among them p65 is one of the most studied protein (Hayden and Ghosh, 2004; Jiang et al., 2016). The activation of signaling may reflect the severity of inflammation to some extent (Liu et al., 2017). Under normal conditions, NF-кB p65 subunit and its inhibitory protein I $\mathrm{B} \alpha$ are in a resting state. Under LPS stimulation, IК $\mathrm{B} \alpha$ is phosphorylated, and P65 is transferred into the nucleus and induces an inflammatory response. MAPK signaling pathway has also been reported to play an essential role in the TLR4-mediated inflammatory response (Lai et al., 2017), and can activate AP-1 and then induce the production of pro-inflammatory cytokines (Ding et al., 2010). Our results showed that magnoflorine remarkably suppressed the phosphorylation of NF- $\kappa \mathrm{B}$ and MAPK in vivo and in vitro.

In summary, our studies indicate that magnoflorine exerts its anti-inflammatory effects by reducing the expression of inflammatory factors in LPS-induced ALI. The possible mechanisms are associated with the inactivation of TLR4mediated NF- $\mathrm{B}$ and MAPK signaling pathways (Figure 9). Importantly, magnoflorine can pass through connection of inflammatory factors and NF- $\mathrm{B}$ and MAPK signaling pathways in vivo and in vitro. Finally, it is hoped that magnoflorine might 
become a potential therapeutic agent for the treatment of LPS-induced ALI.

\section{AUTHOR CONTRIBUTIONS}

SG and KJ conceived and designed the experiments. KJ, CY, and YY carried out the experiments. JY, GZ, and HW analyzed the

\section{REFERENCES}

Akira, S., Hirano, T., Taga, T., and Kishimoto, T. (1990). Biology of multifunctional cytokines: IL 6 and related molecules (IL 1 and TNF). FASEB J. 4, 2860-2867. doi: 10.1096/fasebj.4.11.2199284

Beutz, M. A., and Abraham, E. (2005). Community-acquired pneumonia and sepsis. Clin. Chest Med. 26, 19-28. doi: 10.1016/j.ccm.2004.10.015

Cachofeiro, V., Goicochea, M., de Vinuesa, S. G., Oubiña, P., Lahera, V., and Luño, J. (2008). Oxidative stress and inflammation, a link between chronic kidney disease and cardiovascular disease. Kidney Int. Suppl. 74, S4-S9. doi: 10.1038/ki.2008.516

Chen, T., Xiao, L., Zhu, L., Ma, S., Yan, T., and Ji, H. (2015). Anti-asthmatic effects of ginsenoside rb1 in a mouse model of allergic asthma through relegating Th1/Th2. Inflammation 38, 1814-1822. doi: 10.1007/s10753-015-0159-4

Chiavaroli, A., Brunetti, L., Orlando, G., Recinella, L., Ferrante, C., Leone, S., et al. (2010). Resveratrol inhibits isoprostane production in young and aged rat brain. J. Biol. Regul. Homeost. Agents 24, 441-446.

Cronin, J. G., Kanamarlapudi, V., Thornton, C. A., and Sheldon, I. M. (2016). Signal transducer and activator of transcription-3 licenses toll-like receptor 4dependent interleukin (IL)-6 and IL-8 production via IL-6 receptor-positive feedback in endometrial cells. Mucosal Immunol. 9, 1125-1136. doi: 10.1038/ mi.2015.131

Ding, M., Zhao, J., Bowman, L., Lu, Y., and Shi, X. (2010). Inhibition of AP-1 and MAPK signaling and activation of Nrf2/ARE pathway by quercitrin. Int. J. Oncol. 36, 59-67.

Driver, C. (2012). Pneumonia part 1: pathology, presentation and prevention. Br. J. Nurs. 21, 103-106. doi: 10.12968/bjon.2012.21.2.103

Giebelen, I. A., van Westerloo, D. J., Larosa, G. J., de Vos, A. F., and van der Poll, T. (2007). Local stimulation of alpha7 cholinergic receptors inhibits LPS-induced TNF-alpha release in the mouse lung. Shock 28, 700-703.

Gong, J., Guo, S., Li, H. B., Yuan, S. Y., Shang, Y., and Yao, S. L. (2012). BML-111, a lipoxin receptor agonist, protects haemorrhagic shock-induced acute lung injury in rats. Resuscitation 83, 907-912. doi: $10.1016 /$ j.resuscitation.2011.12.035

Hayden, M. S., and Ghosh, S. (2004). Signaling to NF-кB. Genes Dev. 18, 2195-2224. doi: 10.1101/gad.1228704

Hung, T., Na, M., Min, B., Zhang, X., Lee, I., Ngoc, T., et al. (2007). Protective effect of magnoflorine isolated from coptidis rhizoma on $\mathrm{Cu} 2+$-induced oxidation of human low density lipoprotein. Planta Med. 73, 1281-1284. doi: 10.1055/s2007-981615

Jiang, K., Chen, X., Zhao, G., Wu, H., Mi, J., Qiu, C., et al. (2016). IFN- $\tau$ Plays an anti-inflammatory role in Staphylococcus aureus-induced endometritis in mice through the suppression of NF-KB pathway and MMP9 expression. J. Interferon Cytokine Res. 37, 81-89. doi: 10.1089/jir.2016.0058

Jiang, K., Guo, S., Zhang, T., Yang, Y., Zhao, G., Shaukat, A., et al. (2018). Downregulation of TLR4 by miR-181a Provides negative feedback regulation to lipopolysaccharide-induced inflammation. Front. Pharmacol. 9:142. doi: 10. 3389/fphar.2018.00142

Jiang, K., Ma, X., Guo, S., Zhang, T., Zhao, G., Wu, H., et al. (2017a). Antiinflammatory effects of rosmarinic acid in lipopolysaccharide-induced mastitis in mice. Inflammation 41, 437-448. doi: 10.1007/s10753-017-0700-8

Jiang, K., Zhao, G., Deng, G., Wu, H., Yin, N., Chen, X., et al. (2017b). Polydatin ameliorates Staphylococcus aureus-induced mastitis in mice via inhibiting TLR2-mediated activation of the p38 MAPK/NF- $\mathrm{kB}$ pathway. Acta Pharmacol. Sin. 38, 211-222. doi: 10.1038/aps.2016.123

Klugman, K. P. (1990). Pneumococcal resistance to antibiotics. Clin. Microbiol. Rev. 3, 171-196. doi: 10.1128/CMR.3.2.171 data. SG and GD wrote the manuscript. All authors agree to be responsible for the content of the work.

\section{FUNDING}

This study was supported by the National Natural Science Foundation of China (No. 31772816).

Kuriakose, S., Muleme, H., Onyilagha, C., Okeke, E., and Uzonna, J. E. (2013). Diminazene aceturate (berenil) modulates LPS induced pro-inflammatory cytokine production by inhibiting phosphorylation of MAPKs and STAT proteins. Innate Immun. 20, 760-773. doi: 10.1177/17534259135 07488

Lai, J. L., Liu, Y. H., Liu, C., Qi, M. P., Liu, R. N., Zhu, X. F., et al. (2017). Indirubin inhibits LPS-induced inflammation via TLR4 abrogation mediated by the NF-kB and MAPK signaling pathways. Inflammation 40, 1-12. doi: 10.1007/s10753-016-0447-7

Li, C., and Wang, M. H. (2014). Potential biological activities of magnoflorine: a compound from Aristolochia debilis sieb. et Zucc. Korean J. Plant Res. 27, 223-228. doi: 10.7732/kjpr.2014.27.3.223

Li, W., Fu, K., Lv, X., Wang, Y., Wang, J., Li, H., et al. (2015). Lactoferrin suppresses lipopolysaccharide-induced endometritis in mice via down-regulation of the NF-kB pathway. Int. Immunopharmacol. 28, 695-699. doi: 10.1016/j.intimp. 2015.07.040

Lim, J., Stirling, B., Derry, J., Koga, T., Jono, H., Woo, C., et al. (2007). Tumor suppressor CYLD regulates acute lung injury in lethal Streptococcus pneumoniae infections. Immunity 27, 349-360. doi: 10.1016/j.immuni.2007.07.011

Lin, H. J., Huang, S. S., Huang, G. J., Wu, W. T., Deng, J. S., and Chang, J. S. (2018). Preventive effects of velvet antler (Cervus elaphus) against lipopolysaccharideinduced acute lung injury in mice by inhibiting MAPK/NF-кB activation and inducing AMPK/Nrf2 pathways. Evid. Based Complementary Altern. Med. 2018:2870503.

Liu, T., Zhang, L., Joo, D., and Sun, S. C. (2017). NF-кB signaling in inflammation. Signal Transduct. Target. Ther. 2:17023. doi: 10.1038/sigtrans.2017.23

Livak, K. J., and Schmittgen, T. D. (2001). Analysis of relative gene expression data using real-time quantitative PCR and the 2(-Delta Delta C(T)) Method. Methods 25, 402-408. doi: 10.1006/meth.2001.1262

Lu, Y., Liu, J., Li, H., and Gu, L. (2016). Piperine ameliorates lipopolysaccharideinduced acute lung injury via modulating NF- $\mathrm{KB}$ signaling pathways. Inflammation 39, 303-308. doi: 10.1007/s10753-015-0250-x

Mateu, A., Ramudo, L., Manso, M. A., and De Dios, I. (2015). Cross-talk between TLR4 and PPAR $\gamma$ pathways in the arachidonic acid-induced inflammatory response in pancreatic acini. Int. J. Biochem. Cell Biol. 69, 132-141. doi: 10.1016/ j.biocel.2015.10.022

Matthay, M. A., and Zimmerman, G. A. (2005). Acute lung injury and the acute respiratory distress syndrome: four decades of inquiry into pathogenesis and rational management. Am. J. Respir. Cell Mol. Biol. 33, 319-327. doi: 10.1165/ rcmb.F305

Menghini, L., Ferrante, C., Leporini, L., Recinella, L., Chiavaroli, A., Leone, S., et al. (2016a). A natural formula containing lactoferrin, Equisetum arvensis, soy isoflavones and vitamin D3 modulates bone remodeling and inflammatory markers in young and aged rats. J. Biol. Regul. Homeost. Agents 30, 985-996.

Menghini, L., Ferrante, C., Leporini, L., Recinella, L., Chiavaroli, A., Leone, S., et al. (2016b). An hydroalcoholic chamomile extract modulates inflammatory and immune response in HT29 cells and isolated rat colon. Phytother. Res. 30, 1513-1518. doi: 10.1002/ptr.5655

Nakano, T. (1954). Studies on the alkaloids of magnoliaceous plants. XIII. alkaloids of Magnolia grandiflora L. (2). Pharm. Bull. 2, 326-328. doi: $10.1248 / \mathrm{cpb} 1953.2 .326$

Odobasic, D., Yang, Y., Muljadi, R. C., O'Sullivan, K. M., Kao, W., Smith, M., et al. (2014). Endogenous myeloperoxidase is a mediator of joint inflammation and damage in experimental arthritis. Arthritis Rheumatol. 66, 907-917. doi: 10.1002/art.38299 
Rackovã, L., Májeková, M., Kost'álová, D., and Stefek, M. (2004). Antiradical and antioxidant activities of alkaloids isolated from Mahonia aquifolium. Structural aspects. Bioorg. Med. Chem. 12, 4709-4715. doi: 10.1016/j.bmc.2004. 06.035

Shang, Y., Jiang, Y. X., Ding, Z. J., Shen, A. L., Xu, S. P., Yuan, S. Y., et al. (2010). Valproic acid attenuates the multiple-organ dysfunction in a rat model of septic shock. Chin. Med. J. 123, 2682-2687.

Takeda, K., and Akira, S. (2001). Regulation of innate immune responses by toll-like receptors. Jpn. J. Infect. Dis. 54, 209-219.

Takeda, K., and Akira, S. (2004). TLR signaling pathways. Semin. Immunol. 16, 3-9. doi: 10.1016/j.smim.2003.10.003

Takeuchi, O., and Akira, S. (2010). Pattern recognition receptors and inflammation. Cell 140, 805-820. doi: 10.1016/j.cell.2010.01.022

Treggiari, M. M., Hudson, L. D., Martin, D. P., Weiss, N. S., Caldwell, E., and Rubenfeld, G. (2004). Effect of acute lung injury and acute respiratory distress syndrome on outcome in critically ill trauma patients. Crit. Care Med. 32, 327-331. doi: 10.1097/01.CCM.0000108870. 09693.42

Wang, X., and Quinn, P. J. (2010). Lipopolysaccharide: biosynthetic pathway and structure modification. Prog. Lipid Res. 49, 97-107. doi: 10.1016/j.plipres.2009. 06.002

Wu, H., Gan, Z., Jiang, K., Chen, X., Zhe, Z., Qiu, C., et al. (2016a). Plantamajoside ameliorates lipopolysaccharide-induced acute lung injury via suppressing NF-кB and MAPK activation. Int. Immunopharmacol. 35, 315-322. doi: 10. 1016/j.intimp.2016.04.013

Wu, H., Zhao, G., Jiang, K., Chen, X., Rui, G., Qiu, C., et al. (2016b). IFN- $\tau$ alleviates lipopolysaccharide-induced inflammation by suppressing NF- $\mathrm{B}$ and MAPKs pathway activation in mice. Inflammation 39, 1141-1150. doi: 10.1007/s10753016-0348-9

Wu, H., Zhao, G., Jiang, K., Chen, X., Zhu, Z., Qiu, C., et al. (2016c). Plantamajoside ameliorates lipopolysaccharide-induced acute lung injury via suppressing NF-кB and MAPK activation. Int. Immunopharmacol. 35, 315-322. doi: 10.1016/j.intimp.2016.04.013
Wu, H., Zhao, G., Jiang, K., Li, C., Qiu, C., and Deng, G. (2016d). Engeletin alleviates lipopolysaccharide-induced endometritis in mice by inhibiting TLR4mediated NF-кB activation. J. Agric. Food Chem. 64, 6171-6178. doi: 10.1021/ acs.jafc.6b02304

Wu, X., Xu, W., Feng, X., He, Y., Liu, X., Gao, Y., et al. (2015). TNF-a mediated inflammatory macrophage polarization contributes to the pathogenesis of steroid-induced osteonecrosis in mice. Int. J. Immunopathol. Pharmacol. 28, 351-361. doi: 10.1177/0394632015593228

Xu, X., Yin, P., Wan, C., Chong, X., Liu, M., Cheng, P., et al. (2014). Punicalagin inhibits inflammation in LPS-induced RAW264.7 macrophages via the suppression of TLR4-mediated MAPKs and NF-кB activation. Inflammation 37, 956-965. doi: 10.1007/s10753-014-9816-2

Yang, J., Li, S., Wang, L., Du, F., Zhou, X., Song, Q., et al. (2018). Ginsenoside Rg3 attenuates lipopolysaccharide-induced acute lung injury via MerTK-dependent activation of the PI3K/AKT/mTOR pathway. Front. Pharmacol. 9:850. doi: 10. 3389/fphar.2018.00850

Zhang, X., Li, J., Chen, C., Ci, X., Yu, Q., Zhang, X., et al. (2011). Protective effect of abamectin on acute lung injury induced by lipopolysaccharide in mice. Fundam. Clin. Pharmacol. 25, 700-707. doi: 10.1111/j.1472-8206.2010.00896.x

Conflict of Interest Statement: The authors declare that the research was conducted in the absence of any commercial or financial relationships that could be construed as a potential conflict of interest.

The reviewers, LB and CF, and the handling Editor declared their shared affiliation.

Copyright (C) 2018 Guo, Jiang, Wu, Yang, Yang, Yang, Zhao and Deng. This is an open-access article distributed under the terms of the Creative Commons Attribution License (CC BY). The use, distribution or reproduction in other forums is permitted, provided the original author(s) and the copyright owner(s) are credited and that the original publication in this journal is cited, in accordance with accepted academic practice. No use, distribution or reproduction is permitted which does not comply with these terms. 